\title{
Comparative Study on the Performance of S- Shaped Diffuser With Shorten Length
}

\author{
Raed A. Jessam ${ }^{1,2}$, Hussain H. Al-Kayiem ${ }^{2,}$, and Mohammad S. Nasif ${ }^{1}$ \\ ${ }^{1}$ Mech. Eng. Dept., Universiti Teknologi PETRONAS, 32610 Bandar Seri Iskandar, \\ Malaysia \\ ${ }^{2}$ Electromechanical Eng. Dept., University of Technology, Baghdad, Iraq
}

\begin{abstract}
This study presents numerical investigation on the performance of S-shaped air intake normal and aggressive diffuser with $22 \%$ length reduction. Both models have same area ratio of 3.1 with different total length, turning angle and radius of curvature. The numerical investigation was implemented using CFD simulation by ANSYS-FLUENT 15 software. The inlet Reynold number was $4 \times 10^{4}$ and turbulence intensity $4.1 \%$. The performance evaluation was performed throw evaluation the static pressure coefficient, pressure loss coefficient, distortion coefficient and static pressure wall coefficient. The numerical results show that the performance in the case of aggressive S-shaped diffuser has been reduced compared to the normal S-shaped diffuser. This reduction resulting from the early flow separation and increase of the separation zone due to the high curvature of top and bottom surfaces of aggressive S-shaped diffuser. The results show that the static pressure recovery coefficient decreased by $31 \%$, the total pressure loss coefficient and distortion coefficient increased by $9.5 \%$ and $8.2 \%$, respectively, compared to the S-shaped diffuser. The static pressure wall coefficient on the top and bottom surfaces was dropped with the aggressive S-shaped diffuser.
\end{abstract}

\section{Introduction}

In aero engines, the air intakes represent the main component of breathing of engine. The main function is to maintain high pressure recovery with less inflow to the engine. The performance of aero engines influenced by air intake so much. The S-shaped diffuser has a widely use compared to other types of intakes [1]. Therefore, the study of S-shaped diffuser one of the prevailing research subjects in mechanical engineering because of the important applications for example of aero engines. The size and weight constraints encourage to use shorter S-shaped diffuser. This process lead to aggressive S-shaped diffuser geometry with high diffusion. The ability to shorten the duct is possible by reducing the length but the risk of separation is going up and static pressure losses increase. S-shaped diffusers have increased cross-sectional areas and centerline curvatures with the flow direction. Secondary flows will be increase and cross-stream pressure gradients build up due to centerline curvature. The S-shaped diffuser must be achieving minimal total pressure losses and

\footnotetext{
* Corresponding author : hussain_kayiem@utp.edu.myg
} 
facilitate almost uniform flow with small cross-flow velocity components at the outlet plane to be acceptable [2]. Maren et al [3] applied a new concept to reduce the length of S-duct, called integrated concept (IC), and compared with a base design. This concept represented by replacing the first row of low profile vane from the first bearing engine rotor by struts. The results show that total pressure loss base design is $4.4 \%$ and the total pressure loss with IC is $4.3 \%$.

Other technique has been used by [4] to investigate numerically the effect of employing the energy promoters with different configurations on improving the performance of Sshaped aggressive diffuser. The results show that the static pressure recovery was improved by $47 \%$ and total loss coefficient was reduced by $56 \%$. In the same way, LPVGs at first bend S-shaped have been investigated to study the influence of applying passive flow control on the flow separation [5]. Experimental study on the counter-rotating and co-rotating VGs configurations has been employed on the casing of aggressive interturbine S-duct [6]. The results show that the co-rotating VGs configurations were more active.

The objective of the present study is to investigate the effect of reduce the total length, thereby size reduction, on the performance of annular air intake S-shaped diffuser. The investigation was carried out on both normal and aggressive S-shaped diffusers numerically by CFD three-dimensional 3D simulations utilizing ANSYS-FLUENT 15 software. Static pressure recovery coefficient $C_{P R}$, total pressure loss coefficient $C_{T L}$, static pressure wall coefficient $C_{W P R}$ and distortion coefficient $D C\left(45^{\circ}\right)$ have been adopted as indicators to compare the performance of both diffusers.

\section{Proposed geometry}

Figure 1 shows the proposed geometry of the investigated S-shaped diffuser. The modelled diffusers have area conduit for smooth air inflow with $65 \mathrm{~mm}$ long $\left(b_{i}\right) \times 65 \mathrm{~mm}$ height $\left(l_{i}\right)$ and $65 \mathrm{~mm}$ long $\left(b_{o}\right) \times 125 \mathrm{~mm}$ height $\left(l_{i}\right)$ at the outlet. The proposed geometry of the both models is only $45^{\circ}$ section of the $360^{\circ}$ of the complete annular shape of the diffuser. All the geometrical parameters for both models are summarized in Table 1
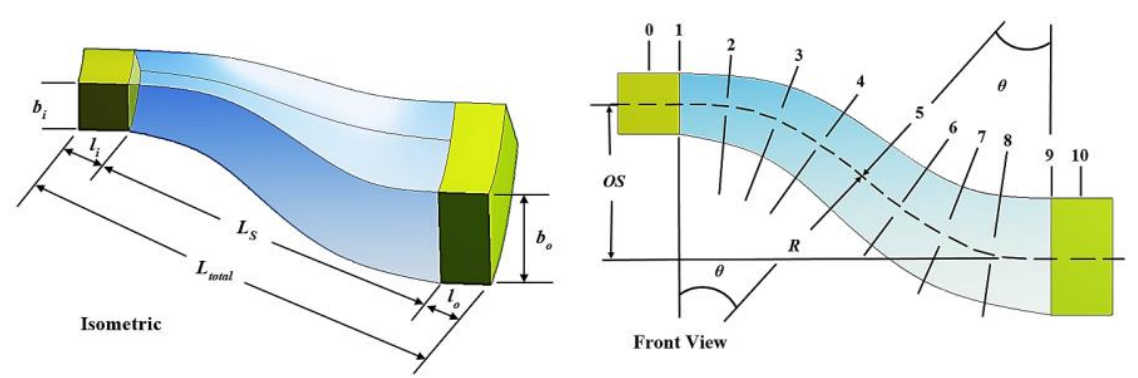

Fig. 1. Geometry of modeled S-shaped diffuser with interesting planes.

Table 1. Geometry specifications of S-shaped diffuser and S-shaped aggressive diffuser models.

\begin{tabular}{|l|c|c|}
\hline \multicolumn{1}{|c|}{ Parameter } & S-shaped diffuser & S-shaped aggressive diffuser \\
\hline Area ratio, $A R(A o / A i)$ & 3.1 & 3.1 \\
\hline Model total length, $L_{\text {total }}$ & $526 \mathrm{~mm}$ & $410 \mathrm{~mm}$ \\
\hline Test section length, $L_{S}$ & $396 \mathrm{~mm}$ & $280 \mathrm{~mm}$ \\
\hline Turning angle, $\theta_{b}$ & $45^{\circ} / 45^{\circ}$ & $35.3^{\circ} / 35.3^{\circ}$ \\
\hline Centreline turning radius, $R$ & $280 \mathrm{~mm}$ & $242.5 \mathrm{~mm}$ \\
\hline
\end{tabular}




\section{Performance indicators}

The important indicators are using for performance evaluation of S-shaped diffuser are:

\subsection{Static pressure recovery coefficient CPR}

It represents the value of kinetic energy that has been converted into pressure energy due to diffusing at any position along the S-shaped diffuser, it can by denoted by the equation:

$$
C_{P R}=\frac{P_{S}-P_{s i}}{\frac{1}{2} \rho U_{a v i}^{2}}
$$

Where: $P_{s}=$ static pressure $\left(\mathrm{N} / \mathrm{m}^{2}\right), P_{s i}=$ inlet static pressure $\left(\mathrm{N} / \mathrm{m}^{2}\right)$ and $U_{a v i}=$ average inlet velocity $(\mathrm{m} / \mathrm{s})$.

\subsection{Total pressure loss coefficient $C_{T L}$}

This indicator represented the value of lost total pressure as a proportion of the mean inlet dynamic pressure due to viscous forces and turbulent mixing, it represented by:

$$
C_{T L}=\frac{P_{t i}-P_{t}}{\frac{1}{2} \rho U_{a v i}^{2}}
$$

Where: $P_{t i}=$ inlet total pressure $\left(\mathrm{N} / \mathrm{m}^{2}\right)$ and $P_{t}=$ total pressure $\left(\mathrm{N} / \mathrm{m}^{2}\right)$.

\subsection{Wall static pressure coefficient $C_{\text {WPR }}$}

It is represented by the following equation:

$$
C_{W P R}=\frac{P_{w s}-P_{w s i}}{\frac{1}{2} \rho U_{a v i}^{2}}
$$

Where: $P_{w s}=$ wall static pressure $\left(\mathrm{N} / \mathrm{m}^{2}\right)$ and $P_{w s i}=$ inlet wall static pressure $\left(\mathrm{N} / \mathrm{m}^{2}\right)$

\subsection{Distortion coefficient $D C\left(45^{\circ}\right)$}

It is represented by the following equation:

$$
D C\left(45^{\circ}\right)=\frac{P_{t \max }-P_{\text {tmino }}}{P_{\text {tavo }}}
$$

Where: $P_{t \min }=$ minimum total pressure at the outlet plane $\left(\mathrm{N} / \mathrm{m}^{2}\right), P_{t \max }=$ maximum total at outlet plane pressure $\left(\mathrm{N} / \mathrm{m}^{2}\right)$ and $P_{\text {tav o }}=$ average total at the outlet plane pressure $\left(\mathrm{N} / \mathrm{m}^{2}\right)$.

\section{Numerical approach}

\subsection{Governing equations}

The governing equations for continuity and momentum, as well as for steady, turbulent, 3D, and incompressible flows of air are as expressed as follows:

The continuity equation in Cartesian coordinates $(\mathrm{x}, \mathrm{y}, \mathrm{z})$ :

$$
\frac{\partial u}{\partial x}+\frac{\partial v}{\partial y}+\frac{\partial w}{\partial z}=0
$$

X-Momentum equation: 


$$
\rho\left(u \frac{\partial u}{\partial x}+v \frac{\partial u}{\partial y}+w \frac{\partial u}{\partial z}\right)=-\frac{\partial P}{\partial x}+\rho g_{x}+\mu\left(\frac{\partial^{2} u}{\partial x^{2}}+\frac{\partial^{2} u}{\partial y^{2}}+\frac{\partial^{2} u}{\partial z^{2}}\right)
$$

Y- Momentum equation:

$$
\rho\left(u \frac{\partial v}{\partial x}+v \frac{\partial v}{\partial y}+w \frac{\partial v}{\partial z}\right)=-\frac{\partial P}{\partial y}+\rho g_{y}+\mu\left(\frac{\partial^{2} v}{\partial x^{2}}+\frac{\partial^{2} v}{\partial y^{2}}+\frac{\partial^{2} v}{\partial z^{2}}\right)
$$

Z- Momentum equation:

$$
\rho\left(u \frac{\partial w}{\partial x}+v \frac{\partial w}{\partial y}+w \frac{\partial w}{\partial z}\right)=-\frac{\partial P}{\partial z}+\rho g_{z}+\mu\left(\frac{\partial^{2} w}{\partial x^{2}}+\frac{\partial^{2} w}{\partial y^{2}}+\frac{\partial^{2} w}{\partial z^{2}}\right)
$$

By solving the equations from (5) to (8) the flow field have been predicated. The 3D Standard $k-\varepsilon$ turbulence model has been adopted for quantities simulation. Near-wall scheme is performed with enhanced wall treatment to address the boundary layer formed during grid generation.

\subsection{Boundary conditions}

The average inlet velocity, inlet pressure and outlet gauge pressure as listed in Table 2 . Diffuser walls considered as no slip boundary condition and all simulation cases were performed with standard air at temperature $30^{\circ} \mathrm{C}$.

Table 2. Description of boundary conditions.

\begin{tabular}{|c|c|c|c|c|c|}
\hline No & Boundary conditions & Value & No & Boundary conditions & Value \\
\hline 1 & Inlet velocity & $9.4(\mathrm{~m} / \mathrm{s})$ & 4 & Wall shear condition & No-slip \\
\hline 2 & Turbulence intensity & $4.1 \%$ & 6 & Air Density $(\rho)$ & $1.165 \mathrm{~kg} / \mathrm{m}^{3}$ \\
\hline 3 & Pressure (outlet) & $0 \mathrm{~Pa}$ & 7 & Air Viscosity $(\mu)$ & $1.85 \times 10^{5} \mathrm{~kg} / \mathrm{m} . \mathrm{s}$ \\
\hline
\end{tabular}

\subsection{Mesh generation and mesh independence}

The geometry of S-shaped diffuser has been modeled by modular design ANSYS 15 and generated by ANSYS - FLUENT 15. To determine the optimum number of mesh cells used to obtain accurate solution, a mesh refinement has been conducted. Mesh independence study based on the $C_{W P R}$ to select the optimum number of elements as in Table 3.

\begin{tabular}{|c|c|c|c|c|c|c|}
\hline \multirow{3}{*}{$\begin{array}{c}\text { No. } \\
\text { of elements }\end{array}$} & \multicolumn{6}{|c|}{ Static Pressure Wall Coefficient $C_{W P R}$} \\
\hline & \multicolumn{2}{|c|}{ Top surface } & \multirow{2}{*}{ Deviation } & \multicolumn{2}{|c|}{ Bottom surface } & \multirow{2}{*}{ Deviation } \\
\hline & Exp. & CFD & & Exp. & CFD & \\
\hline 183402 & \multirow{4}{*}{0.759} & 0.931 & $23 \%$ & \multirow{4}{*}{0.795} & 0.958 & $21 \%$ \\
\hline 214417 & & 0.893 & $18 \%$ & & 0.926 & $16.5 \%$ \\
\hline 666976 & & 0.856 & $13 \%$ & & 0.901 & $13.3 \%$ \\
\hline 843827 & & 0.825 & $9.2 \%$ & & 0.864 & $9.5 \%$ \\
\hline
\end{tabular}

Table 3. Static pressure wall coefficient $C_{W P R}$ variation with refining the grid of S-shaped aggressive diffuser compared to the experimental case.

It is obvious with the finer mesh 843827 elements the results were very close with previous experiment case of $9.2 \%$ and $8.6 \%$ for the top and bottom surfaces, respectively. Therefore, 843827 elements are used as the optimum number of mesh for the simulation. 


\section{Results and discussion}

The numerical results show that the $C_{P R}$ has been reduced by $31 \%$. This drop created from the high bending surface of diffuser which is accelerate the flow especially on the bottom surface, thereby increase the flow separation and reduce the static pressure of aggressive $S$ shaped diffuser. Also, the $C_{T L}$ was increased by $9.5 \%$ with aggressive diffuser. Fig. 2 explains the distribution of velocity vectors and contours at the outlet plane for both diffusers and it can observe higher velocity with aggressive diffuser and cover larger area Fig.2-A compared to S-shaped diffuser Fig.2-B. Also, strong counter-rotating vortices, with bounded circular area, on the corners of the bottom surface of aggressive $\mathrm{S}$-shaped diffuser were generated. This process leading reduces $C_{P R}$ and increase the $C_{T L}$ at the outlet plane.

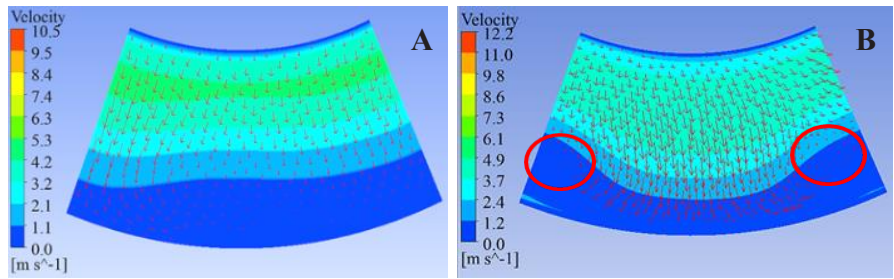

Fig. 2. Velocity vectors and velocity contours at the outlet plane of: A-normal S-shaped diffuser and B- aggressive S-shaped diffuser.

The other important indicator used for performance evaluation is $D C\left(45^{\circ}\right)$. The total pressure values with measured data and numerical results of aggressive diffuser was higher, also, the difference between the maximum and minimum values was large compared with values of normal diffuser, as shown in Fig.3-A and 3-B. This large difference increases the $D C\left(45^{\circ}\right)$ at the outlet plane and reduces the flow uniformity at the outlet plane. The $D C\left(45^{\circ}\right)$ was increased by $8.2 \%$ of the aggressive diffuser compared to normal diffuser as explain in Table 4.
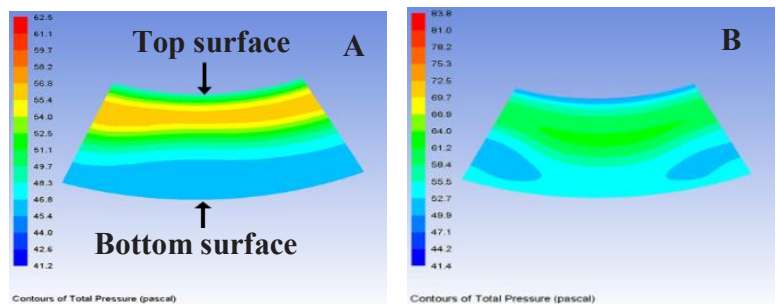

Fig. 3. Total pressure contours at the outlet plane of S-shaped diffuser: A. normal B. aggressive.

Table 4. Performance parameters of S-shaped diffuser and S-shaped aggressive diffuser models.

\begin{tabular}{|c|c|c|}
\hline Performance Parameter & S-shaped diffuser & S-shaped aggressive diffuser \\
\hline$C_{P R}$ & 0.76 & 0.58 \\
\hline$C_{T L}$ & 0.114 & 0.126 \\
\hline$D C(45)^{\circ}$ & 0.168 & 0.183 \\
\hline
\end{tabular}

Fig. 4 shows the results of $C_{W P R}$ along the length of the normal diffuser and aggressive diffuser. In both diffusers, the bottom surfaces have an accelerated flow resulting from high diffusion due to the strong curvature at the first bend. The process drops the $C_{W P R}$ to minimum value. After this drop, the $C_{W P R}$ recovered by the deceleration due to increase in the crosssectional area. The reduction in $C_{W P R}$ with aggressive S-shaped diffuser is less than -0.2 
compared to normal S-shaped diffuser -0.17 . Also, the flow separation started at $X \approx 66 \mathrm{~mm}$ of aggressive diffuser and at $X \approx 135 \mathrm{~mm}$ of normal diffuser. After this reduction, the $C_{W P R}$ starts recover until the outlet. For the top surface, the $C_{W P R}$ was increased in the initial phase 0.61 and 0.69 up to plane 3 around $X \approx 90 \mathrm{~mm}$ and $X \approx 125 \mathrm{~mm}$ for normal and aggressive diffuser, respectively. After that the $C_{W P R}$ decreased by the accelerated flow until plane 7 at $X \approx 200 \mathrm{~mm}$ and $X \approx 270 \mathrm{~mm}$ for normal and aggressive diffusers, respectively, when the flow reattached again and then it increases steadily till the outlet plane.

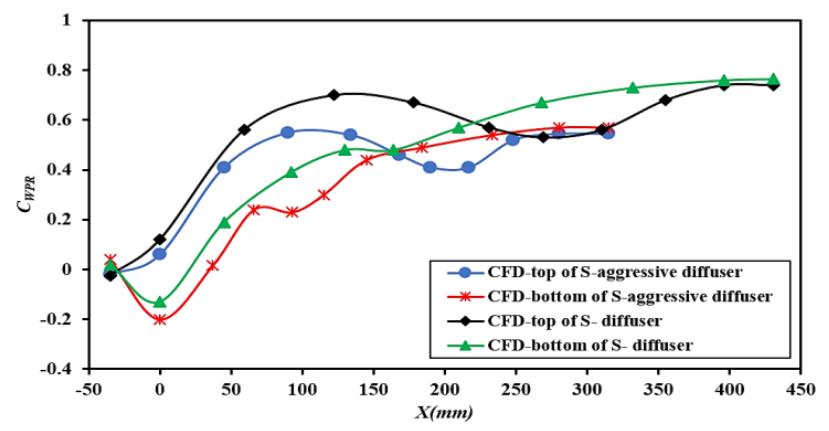

Fig. 4. Numerical Results of $C_{W P R}$ along the length of normal S-shaped diffuser and aggressive Sshaped diffuser.

\section{Conclusions}

Numerical investigations have been conducted on normal S-shaped diffuser and aggressive $\mathrm{S}$-shaped diffuser. The results indicated that reduction the total length of normal S-shaped diffuser to create a diffuser which has been named aggressive S-shaped diffuse resulting in a reduction in $C_{P R}$ about $31 \%$, increase the $C_{T L}$ by $9.5 \%$ and $D C\left(45^{\circ}\right)$ by $8.2 \%$. The flow separation was started early and the size of separation zone was large with aggressive Sshaped diffuser. This process leading to reduction in $C_{W P R}$.

The authors acknowledge Universiti Teknologi PETRONAS (UTP) - Malaysia for the logistic and technical support to produce this work. The first author appreciates UTP for the financial support to produce the paper under YUTP grant 0153AA-E61.

\section{References}

1. A. Madadi, M. J. Kermani, and M. Nili-Ahmadabadi. Journal of Engineering for Gas Turbines and Power 136, no. 12, (2014).

2. Paul, A. R., Ranjan, P., Patel, V. K. and Jain, A. Aerospace Science and Technology 28, no. 1, 332-343. (2013).

3. Marn, A., E. GÃksttlich, D. Cadrecha, and H. P. Pirker. Journal of turbomachinery 131, no. 4, 041014, (2009).

4. R. A. Jessam, H.H. Al-Kayiem and M .S. Nasif, 2017. ARPN Journal of Engineering and Applied Sciences 12, no. 7, (2017).

5. Santner, C., GÃksttlich, E., Marn, A., Hubinka, J. and Paradiso, B. Journal of Turbomachinery 134, no.1, 011023. (2012).

6. Y. Zhang, S. Hu, X. F. Zhang, M. Benner, A. Mahallati, and Vlasic, E. Journal of Engineering for Gas Turbines and Power 136, no. 11, 112604. (2014). 